PROCEEDINGS OF THE AMERICAN MATHEMATICAL SOCIETY

Volume 124, Number 1, January 1996

\title{
LIMITS OF DIFFERENTIABLE FUNCTIONS
}

\author{
UDAYAN B. DARJI
}

(Communicated by J. Marshall Ash)

\begin{abstract}
Suppose that $\left\{f_{n}\right\}$ is a sequence of differentiable functions defined on $[0,1]$ which converges uniformly to some differentiable function $f$, and $\left\{f_{n}^{\prime}\right\}$ converges pointwise to some function $g$. Let $M=\left\{x: f^{\prime}(x) \neq g(x)\right\}$. In this paper we characterize such sets $M$ under various hypotheses. It follows from one of our characterizations that $M$ can be the entire interval $[0,1]$.
\end{abstract}

\section{INTRODUCTION}

We say that a sequence of differentiable functions $\left\{f_{n}\right\}$ defined on the interval $[0,1]$ is proper if $\left\{f_{n}\right\}$ converges uniformly to some differentiable function $f$ and $\left\{f_{n}^{\prime}\right\}$ converges pointwise to some function $g$. For such proper $\left\{f_{n}\right\}$, we let $\Delta\left(\left\{f_{n}\right\}\right)=$ $\left\{x: f^{\prime}(x) \neq g(x)\right\}$. It is a standard theorem in elementary analysis texts [6] that if $\left\{f_{n}\right\}$ is proper and $\left\{f_{n}^{\prime}\right\}$ converges uniformly to some function $g$, then $\Delta\left(\left\{f_{n}\right\}\right)=\emptyset$. It is rather easy to construct an example of a proper $\left\{f_{n}\right\}$ where $\Delta\left(\left\{f_{n}\right\}\right) \neq \emptyset$. In this paper we investigate the following questions:

Question 1. Is there a proper $\left\{f_{n}\right\}$ such that $\Delta\left(\left\{f_{n}\right\}\right)=[0,1]$ ?

Question 2. Can $\left\{\Delta\left(\left\{f_{n}\right\}\right):\left\{f_{n}\right\}\right.$ is proper $\}$ be characterized?

Theorems 1, 2, 3 and 5 answer Question 2 under various hypotheses. It will follow from Theorem 2 that Question 1 has an affirmative answer. However, Theorem 4 implies that in order to make $\Delta\left(\left\{f_{n}\right\}\right)=[0,1]$, the derivatives have to be complicated in some sense.

We now state some definitions and background theorems. Recall that the density topology $\mathcal{D}$ on $\mathbb{R}$ is

$\{M \subset \mathbb{R}: M$ is Lebesgue measurable and has density 1 at each of its points $\}$.

Sets in $\mathcal{D}$ are said to be open in density topology. Whenever we say that a set is open, closed, $G_{\delta}, F_{\sigma}$, etc., we mean that it is open, closed, $G_{\delta}, F_{\sigma}$, etc. in the ordinary topology on $\mathbb{R}$. Whenever we want a set to be open or closed in the density topology, we will specifically so state. A function $f:[0,1] \rightarrow \mathbb{R}$ is approximately

Received by the editors April 20, 1994 and, in revised form, July 6, 1994; this paper was presented at the Special Session on Real Analysis at the 1992 AMS meeting in Baltimore.

1991 Mathematics Subject Classification. Primary 26A24, 26A21; Secondary 40A30.

Key words and phrases. $F_{\sigma}, G_{\delta \sigma}$, density topology, approximate continuity, nowhere measure dense.

This is the core part of the author's dissertation which was directed by Professor Jack B. Brown.

(c)1996 American Mathematical Society 
continuous iff the preimage of every open set under $f$ is open in the density topology. A set $M \subset[0,1]$ is nowhere measure dense in the interval $J$ iff the interior of $M \cap J$ in the density topology is nowhere dense in $J$ in the ordinary topology. We will freely use the following facts about density topology, approximate continuity and Lebesgue integration theory throughout the paper. Their proofs may be found in [1], [3], [2].

Fact 1. If $M \subset \mathbb{R}$ is measurable, then there exits an $F_{\sigma}$ set $N \subset M$ such that $N$ is open in the density topology and $\lambda(M \backslash N)$, the Lebesgue measure of $M \backslash N$, is zero.

Fact 2. Every bounded approximately continuous function is a derivative.

Fact 3 (Zahorski's Theorem [7]). If $G_{0}$ and $G_{1}$ are two disjoint $G_{\delta}$ sets which are closed in the density topology, then there exists an approximately continuous function $f:[0,1] \rightarrow[0,1]$ such that $f^{-1}(i)=G_{i}$ for $i=0,1$.

Fact 4 . If $g$ is an integrable derivative defined on $[0,1]$, then $f(x)=\int_{0}^{x} g$ is differentiable everywhere and $f^{\prime}(x)=g(x)$ for all $x \in[0,1]$.

Fact 5. Suppose $\left\{g_{n}\right\}$ is a sequence of integrable functions defined on the interval $[0,1]$ such that $\left\{g_{n}\right\}$ is dominated by an $L^{1}$ function and $\left\{g_{n}\right\}$ converges pointwise to $g$. Let $f_{n}(x)=\int_{0}^{x} g_{n}$ and $f(x)=\int_{0}^{x} g$ for each $x \in[0,1]$. Then $\left\{f_{n}\right\}$ converges uniformly to $f$.

\section{MAIN RESUlts}

Lemma 1. If $\left\{f_{n}\right\}$ is proper and $\left\{f_{n}^{\prime}\right\}$ is dominated by an $L^{1}$ function, then $\Delta\left(\left\{f_{n}\right\}\right)$ has measure zero.

Proof. Without loss of generality assume that $f_{n}(0)=0$ for all $n$. Let $g$ be the pointwise limit of $\left\{f_{n}^{\prime}\right\}$ and $h(x)=\int_{0}^{x} g$. Note that $h^{\prime}(x)=g(x)$ for almost all $x \in[0,1]$. By the Lebesgue Dominated Convergence Theorem we have that

$$
f(x)=\lim _{n \rightarrow \infty} f_{n}(x)=h(x) \quad \text { for all } x \in[0,1] .
$$

Thus $f^{\prime}=g$ a.e. and $\Delta\left(\left\{f_{n}\right\}\right)$ has measure zero.

Lemma 2. Suppose $\left\{f_{n}\right\}$ is proper. Then $\Delta\left(\left\{f_{n}\right\}\right)$ is $G_{\delta \sigma}$.

Proof. Let $g$ be the pointwise limit of $\left\{f_{n}^{\prime}\right\}$. Since derivatives are of Baire class 1, $g$ is in Baire class 2 and hence $\Delta\left(\left\{f_{n}\right\}\right)=\left(f^{\prime}-g\right)^{-1}(R \backslash\{0\})$ is $G_{\delta \sigma}[4]$.

Theorem 1 (General Dominated Case). A set $M \subset[0,1]$ is $G_{\delta \sigma}$ and of measure zero iff $M=\Delta\left(\left\{f_{n}\right\}\right)$ for some proper $\left\{f_{n}\right\}$ where $\left\{f_{n}^{\prime}\right\}$ is dominated by an $L^{1}$ function.

Proof. $(\Leftarrow)$ This direction follows from Lemmas 1 and 2 .

$(\Rightarrow)$ The proof of this direction has a flavor similar to a result of Preiss [5]. Let $M=\bigcup G_{k}$ be a $G_{\delta \sigma}$ set of measure zero where each $G_{k}$ is $G_{\delta}$. Now for each positive integer $k$, let $\left\{U_{k, n}\right\}_{n=1}^{\infty}$ be a decreasing sequence of open sets such that $G_{k}=$ $\bigcap_{n=1}^{\infty} U_{k, n}$. For each $n$ and $k$ we may obtain by Fact 3 an approximately continuous function $h_{k, n}:[0,1] \rightarrow[0,1]$ such that $h_{k, n}^{-1}(1)=G_{k}$ and $h_{k, n}^{-1}(0)=\left(U_{k, n}\right)^{c}$, the complement of $U_{k, n}$. Note that for each $k,\left\{h_{k, n}\right\}_{n=1}^{\infty}$ converges pointwise to $\chi_{G_{k}}$, 
the characteristic function of $G_{k}$. Now set

$$
g_{n}=\sum_{k=1}^{\infty} \frac{1}{2^{k}} h_{k, n} \quad \text { and } \quad g=\sum_{k=1}^{\infty} \frac{1}{2^{k}} \chi_{G_{k}} .
$$

It follows that $g_{n}$ is a bounded derivative because it is the uniform sum of a sequence of bounded approximately continuous functions. Also note that $\left\{g_{n}\right\}$ converges pointwise to $g$.

Now let $f_{n}(x)=\int_{0}^{x} g_{n}$. That $f_{n}^{\prime}(x)=g_{n}(x)$ for all $x \in[0,1]$ follows from Fact 4 . We also have that $\int_{0}^{x} g=0$ for all $x \in[0,1]$ as $g$ is nonzero only on the measure zero set $M$. Since $\left\{g_{n}\right\}$ is a uniformly bounded sequence of integrable functions which converges pointwise to $g$, by Fact 5 it follows that $\left\{f_{n}\right\}$ converges uniformly to the zero function. But $\left\{f_{n}^{\prime}\right\}$ converges pointwise to the function $g$ which is nonzero precisely on set $M$. Therefore, $\Delta\left(\left\{f_{n}\right\}\right)=M$.

Lemma 3. Let $M \subset[0,1]$ be a nonempty $F_{\sigma}$ set which is open in the density topology, and let $A>0$. Then, there exists a bounded approximately continuous function $f:[0,1] \rightarrow \mathbb{R}$ such that

(1) $\int_{0}^{1} f=A$,

(2) $f^{-1}(0)=M^{c}$, and

(3) $f \geq 0$.

Proof. By Fact 3 there is a bounded approximately continuous function $h$ which satisifies conditions (2) and (3). Let $f=c \cdot h$, where $c=\frac{A}{\int_{0}^{1} h}$. This $f$ satisfies all three required conditions.

Lemma 4. If $M$ is $F_{\sigma}$, open in the density topology and $M^{c}$ is dense in $[0,1]$, then there exists proper $\left\{f_{n}\right\}$ such that $M=\Delta\left(\left\{f_{n}\right\}\right)$.

Proof. Suppose $M$ is $F_{\sigma}$, open in the density topology, and $M^{c}$ is dense in $[0,1]$. Let $g$ be an approximately continuous function such that $0 \leq g(x) \leq 1$ for all $x$ and $g^{-1}(0)=M^{c}$. Write $M=\bigcup_{i=1}^{\infty} F_{i}$ where each $F_{i}$ is a nowhere dense closed subset of $[0,1]$. Since each $F_{i}$ is a nowhere dense closed subset of $[0,1]$ and $M$ is open in the density topology, it follows that for each interval $J$ for which $M \cap J \neq \emptyset$ we have $(M \cap J) \backslash\left(\bigcup_{i=1}^{n} F_{i}\right) \neq \emptyset$ for all $n$.

We now construct a sequence of derivatives $\left\{g_{n}\right\}$ in the following fashion. Fix $n$. Let $N_{n}=M^{c} \cup F_{1} \cup \ldots \cup F_{n}$. Observe that $N_{n}$ is a $G_{\delta}$ set and is also closed in the density topology. Let $\{x(n, k)\}_{k=1}^{m(n)+1}$ be a partition of $[0,1]$ such that $x(n, i) \in M^{c}$ and $|x(n, i)-x(n, i+1)|<\frac{1}{2^{n}}$ for each $1 \leq i \leq m(n)$. Fix $1 \leq i \leq m(n)$. Observe that $[x(n, i), x(n, i+1)] \backslash N_{n}$ is either a nonempty $F_{\sigma}$ set which is open in the density topology or $[x(n, i), x(n, i+1)] \subset M^{c}$. If the latter is the case, let $h_{n, i}$ be the zero function on $[x(n, i), x(n, i+1)]$ and we have that $\int_{x(n, i)}^{x(n, i+1)} h_{n, i}=\int_{x(n, i)}^{x(n, i+1)} g$. Otherwise using Lemma 3, obtain a bounded, nonnegative approximately continuous function $h_{n, i}$ defined on $[x(n, i), x(n, i+1)]$ such that $h_{n, i}^{-1}(0)=N_{n} \cap[x(i), x(i+1)]$ and $\int_{x(n, i)}^{x(n, i+1)} h_{n, i}=\int_{x(n, i)}^{x(n, i+1)} g$. Now, let $h_{n}$ be the union of $h_{n, 1}, \ldots, h_{n, m(n)}$. Then, $h_{n}$ is bounded and approximately continuous. Let $g_{n}=g-h_{n}$. As $\left\{h_{n}\right\}$ converges pointwise to the zero function, $\left\{g_{n}\right\}$ converges pointwise to $g$.

Let $f_{n}(x)=\int_{0}^{x} g_{n}$. Since $g_{n}$ is bounded and approximately continuous, $f_{n}^{\prime}=g_{n}$. Let us next show that $\left\|f_{n}\right\|$, the sup norm of $f_{n}$, is less than $2^{-n+1}$. Let $x \in[0,1]$ 
and $i$ be such that $x \in[x(n, i), x(n, i+1)]$. Then

$$
\begin{aligned}
\left|f_{n}(x)\right|=\left|\int_{0}^{x} g_{n}\right| \leq\left|\int_{0}^{x(n, i)} g-h_{n}\right|+\left|\int_{x(n, i)}^{x} g-h_{n}\right| \\
\leq 0+\left|\int_{x(n, i)}^{x} g\right|+\left|\int_{x(n, i)}^{x} h_{n}\right|<2^{-n}+2^{-n}=2^{-n+1} .
\end{aligned}
$$

The above estimate on $\left\|f_{n}\right\|$ implies that $\left\{f_{n}\right\}$ converges uniformly to the zero function. We also know that $\left\{g_{n}\right\}$ converges pointwise to $g$ and $g^{-1}(0)=M^{c}$. Therefore, $M=\Delta\left(\left\{f_{n}\right\}\right)$.

Theorem 2 (General Nondominated Case). A set $M \subset[0,1]$ is $G_{\delta \sigma}$ iff there exists proper $\left\{f_{n}\right\}$ such that $M=\Delta\left(\left\{f_{n}\right\}\right)$.

Proof. $(\Leftarrow)$ This direction follows from Lemma 2 .

$(\Rightarrow)$ Let $M$ be $G_{\delta \sigma}$. From Fact 1 obtain two disjoint sets $M_{1}$ and $M_{2}$ such that $M_{1} \cup M_{2}=M, M_{1}$ is $G_{\delta \sigma}$ set of measure zero and $M_{2}$ is an $F_{\sigma}$ set which is open in the density topology and $M_{2}^{c}$ is dense in [0,1]. By Theorem 1 and Lemma 4, obtain proper sequences $\left\{f_{n}\right\}$ and $\left\{h_{n}\right\}$ such that $M_{1}=\Delta\left(\left\{f_{n}\right\}\right)$ and $M_{2}=\Delta\left(\left\{h_{n}\right\}\right)$. Then $\left\{f_{n}+h_{n}\right\}$ is proper and $M=\Delta\left(\left\{f_{n}+h_{n}\right\}\right)$.

Lemma 5. Suppose $\left\{f_{n}\right\}$ is proper and for all $n, f_{n} \in C^{1}$, i.e. $f_{n}^{\prime}$ is continuous. Then $\Delta\left(\left\{f_{n}\right\}\right)$ is $F_{\sigma}$.

Proof. Let $g$ be the pointwise limit of $\left\{f_{n}^{\prime}\right\}$. Then $g$ and $f^{\prime}$ are of Baire class 1 . Therefore, $\Delta\left(\left\{f_{n}\right\}\right)=\left(f^{\prime}-g\right)^{-1}(\mathbb{R} \backslash\{0\})$ is $F_{\sigma}[4]$.

Theorem 3 (Dominated $C^{1}$ Case). A set $M \subset[0,1]$ is $F_{\sigma}$ and of measure zero iff $M=\Delta\left(\left\{f_{n}\right\}\right)$ for some proper $\left\{f_{n}\right\}$ where $f_{n} \in C^{1}$ for all $n$ and $\left\{f_{n}^{\prime}\right\}$ is dominated by an $L^{1}$ function.

Proof. $(\Leftarrow)$ This direction follows from Lemmas 1 and 5 .

$(\Rightarrow)$ Suppose $M$ is $F_{\sigma}$ and of measure zero. Let $M=\bigcup_{k=1}^{\infty} M_{k}$ where each $M_{k}$ is closed. Let $\left\{G_{k, n}\right\}$ be such that each $G_{k, n}$ is a finite collection of closed intervals and

(1) $\bigcup G_{k, n} \subset \bigcup G_{k, n+1}$, and

(2) $\bigcup_{n=1}^{\infty} G_{k, n}=M_{k}^{c}$.

Now let $h_{k, n}$ be a continuous function defined on $[0,1]$ such that $0 \leq h_{k, n}(x) \leq 1$, $h_{k, n}\left(M_{k}\right)=1$, and $h_{k, n}\left(\bigcup G_{k, n}\right)=0$. Note that $\left\{h_{k, n}\right\}_{n=1}^{\infty}$ converges pointwise to $\chi_{M_{k}}$, the characteristic function of $M_{k}$. Now set $g_{n}=\sum_{k=1}^{\infty} 2^{-k} \cdot h_{k, n}$ and $g=$ $\sum_{k=1}^{\infty} 2^{-k} \cdot \chi_{M_{k}}$. Observe that $g_{n}$ is a continuous function for all $n, 0 \leq g_{n}(x) \leq 1$ and $\left\{g_{n}\right\}$ converges pointwise to $g$.

Setting $f_{n}(x)=\int_{0}^{x} g_{n}$, we have that $f_{n}^{\prime}(x)=g_{n}(x)$ for all $x \in[0,1]$. We also have that $\int_{0}^{x} g=0$ for all $x \in[0,1]$ as $g$ is nonzero only on the measure zero set $M$. Since $\left\{g_{n}\right\}$ is a uniformly bounded sequence of continuous functions which converges pointwise to $g$, by Fact 5 it follows that $\left\{f_{n}\right\}$ converges uniformly to the zero function. But $\left\{f_{n}^{\prime}\right\}$ converges pointwise to function $g$ which is nonzero precisely on set $M$. Therefore, $\Delta\left(\left\{f_{n}\right\}\right)=M$. 
Lemma 6. Suppose $M \subset[0,1]$ is $F_{\sigma}$ and nowhere measure dense. Then $M$ is the union of two disjoint $F_{\sigma}$ sets, one of which is of measure zero and the other nowhere dense.

Proof. Let $B_{1}, B_{2}, \ldots$ be a countable basis for [0,1]. Let $U=\bigcup\left\{B_{i}: \lambda\left(B_{i} \cap M\right)=\right.$ $0\}$. Since $M$ is nowhere measure dense, $U$ is a dense open subset of $[0,1]$. Let $M_{1}=M \cap U$ and $M_{2}=M \backslash U$. Then $M_{1}$ and $M_{2}$ are the desired sets.

Lemma 7. Let $\left\{g_{n}\right\}$ be a sequence of a.e. continuous functions whose domain is interval I. Suppose $\left\{g_{n}\right\}$ converges pointwise to some function $g$ on $I$. Then there exists an interval $J \subset I$ and $K>0$, such that for all $n,\left|g_{n}(x)\right|<K$ for a.e. $x \in J$.

Proof. We will prove this lemma by contradiction. Assume the hypothesis and that there is no $J \subset I$ such that $\left\{g_{n}\right\}$ is bounded a.e. on $J$. This implies that for every $K>0$ and interval $J \subset I$, there are infinitely many integers $n$ such that $\left|g_{n}\right|>K$ on a positive measure set contained in $J$. Using this observation and the fact that $\left\{g_{n}\right\}$ is a sequence of a.e. continuous functions, we may obtain a subinterval $I_{1}$ of $I$ and a positive integer $n(1)$ such that $\left|g_{n(1)}\right|>1$ on $I_{1}$. Proceeding in a similar fashion we may obtain a decreasing sequence of closed intervals $\left\{I_{k}\right\}$ and an increasing sequence of positive integers $\{n(k)\}$ such that for each $k,\left|g_{n(k)}\right|>k$ on $I_{k}$. Now let $p \in \bigcap_{i=1}^{\infty} I_{k}$. Then $\left\{g_{n(k)}(p)\right\}_{k=1}^{\infty}$ does not converge, contradicting the hypothesis and concluding the proof of the lemma.

Theorem 4. Suppose $\left\{f_{n}\right\}$ is proper, $f_{n}^{\prime}$ is integrable and the set of discontinuities of $f_{n}^{\prime}$ has measure zero for all $n$. Then $\Delta\left(\left\{f_{n}\right\}\right)$ is nowhere measure dense.

Proof. Let $I$ be a subinterval of $[0,1]$. Using Lemma 7 obtain an interval $J \subset I$ such that $\left\{f_{n}^{\prime}\right\}$ is bounded a.e. on $J$. By Lemma 1 we have that $\Delta\left(\left\{f_{n}\right\}\right) \cap J$ has measure zero. This implies that the interior of $\Delta\left(\left\{f_{n}\right\}\right)$ in the density topology is nowhere dense in $[0,1]$.

Lemma 8. Suppose $M$ is an $F_{\sigma}$ nowhere dense subset of $[0,1]$. Then $M=\Delta\left(\left\{f_{n}\right\}\right)$ for some proper $\left\{f_{n}\right\}$ where $f_{n} \in C^{1}$ for all $n$.

Proof. Let $U=(\operatorname{cl}(M))^{c}$ and $F_{1}, F_{2}, \ldots$ be a pairwise disjoint decomposition of $M$ into closed sets. Let $g$ be the function which is zero on $M^{c}$ and $2^{-i}$ on $F_{i}$. Note that $g$ is of Baire class 1. Let $\left\{G_{n}\right\}$ be such that each $G_{n}$ is a finite collection of closed intervals and

- $\bigcup G_{n} \subset \bigcup G_{n+1}$, and

- $\bigcup_{n=1}^{\infty}\left(\bigcup G_{n}\right)=U$.

Using the fact that $g$ is of Baire class 1, obtain a sequence of continuous functions $\left\{h_{n}\right\}$ such that $\left\{h_{n}\right\}$ converges pointwise to $g$, and for all $\left.n, h_{n}\left(\bigcup G_{n}\right)\right)=0$ and $0 \leq h_{n}(x) \leq 1$.

We now construct $g_{n}$ in the following manner. First, let $\{x(n, k)\}_{k=1}^{m(n)+1}$ be a partition of $[0,1]$ such that $|x(n, k)-x(n, k+1)|<2^{-n}$ for $k=1,2, \ldots, m(n)$. Now for each $1 \leq k \leq m(n)$, let $a_{n, k}$ be a continuous nonnegative function defined on $[x(n, k), x(n, k+1)]$ such that

- $\int_{x(n, k)}^{x(n, k+1)} a_{n, k}=\int_{x(n, k)}^{x(n, k+1)} h_{n}$,

- $a_{n, k}=0$ on $\bigcup G_{n} \cap[x(n, k), x(n, k+1)]$,

- $a_{n, k}^{-1}(\mathbb{R} \backslash\{0\}) \subset U$, and

- $a_{n, k}(x(n, k))=a_{n, k}(x(n, k+1))=0$. 
Let $a_{n}$ be the union of $a_{n, 1}, a_{n, 2}, \ldots, a_{n, m(n)}$ and $g_{n}=h_{n}-a_{n}$. As $\left\{a_{n}\right\}$ is a sequence of continuous functions which converges pointwise to the zero function, $\left\{g_{n}\right\}$ is a sequence of continuous functions which converges pointwise to $g$.

For each $n$, let $f_{n}(x)=\int_{0}^{x} g_{n}$. Observe that for $1 \leq k \leq m(n)+1$

$$
f_{n}(x(n, k))=\int_{0}^{x(n, k)} g_{n}=\sum_{i=1}^{k-1} \int_{x(n, i)}^{x(n, i+1)} h_{n}-a_{n}=0 .
$$

Using this observation we obtain an estimate on $\left\|f_{n}\right\|$. Let $x \in[0,1]$. Let $k$ be a positive integer such that $x \in[x(n, k), x(n, k+1)]$.

$$
\begin{aligned}
&\left|f_{n}(x)\right| \leq\left|f_{n}(x(n, k))\right|+\left|f_{n}(x)-f_{n}(x(n, k))\right| \\
&=0+\left|\int_{x(n, k)}^{x} g_{n}\right|=\left|\int_{x(n, k)}^{x} h_{n}-a_{n}\right| \\
& \leq\left|\int_{x(n, k)}^{x} h_{n}\right|+\left|\int_{x(n, k)}^{x} a_{n}\right| \\
&<2^{-n}+2^{-n}=2^{-n+1} .
\end{aligned}
$$

From above we have that $\left\{f_{n}\right\}$ converges uniformly to the zero function. As $\left\{f_{n}^{\prime}\right\}$ converges pointwise to $g$ and $g^{-1}(0)=M^{c}$, we have that $M=\Delta\left(\left\{f_{n}\right\}\right)$.

Theorem 5 (Nondominated $C^{1}$ Case). A set $M \subset[0,1]$ is $F_{\sigma}$ and nowhere measure dense iff $M=\Delta\left(\left\{f_{n}\right\}\right)$ for some proper $\left\{f_{n}\right\}$ where $f_{n} \in C^{1}$ for all $n$.

Proof. $(\Leftarrow)$ This direction follows from Theorem 4 and Lemma 5.

$(\Rightarrow)$ Suppose $M$ is $F_{\sigma}$ and nowhere measure dense. Then by Lemma $6, M=$ $M_{1} \cup M_{2}$ where $M_{1}$ and $M_{2}$ are disjoint $F_{\sigma}$ sets, one of which is nowhere dense and the other of measure zero. By Theorem 3 and Lemma 8 , there are proper sequences $\left\{f_{n}\right\}$ and $\left\{h_{n}\right\}$ such that $\Delta\left(\left\{f_{n}\right\}\right)=M_{1}$ and $\Delta\left(\left\{h_{n}\right\}\right)=M_{2}$. Then $\left\{f_{n}+h_{n}\right\}$ is proper and $M=\Delta\left(\left\{f_{n}+h_{n}\right\}\right)$.

The author thanks the referee for making helpful suggestions which improved the exposition of the paper.

\section{REFERENCES}

1. A. M. Bruckner, Differentiation of real functions, Lecture Notes in Math., vol. 659, SpringerVerlag, New York, 1978. MR 80h:26002

2. K. Ciesielski, L. Larson, and K. Ostaszewski, I-Denstiy continuous functions, Mem. Amer. Math. Soc., no. 515, vol. 107, Amer. Math. Soc., Providence, RI, 1994. MR 94f:54035

3. R. Jeffery, The theory of function of a real variable, Mathematical Exposition No. 6, University of Toronto Press, Ontario, 1951. MR 13:2166

4. K. Kuratowski, Topology, Vol. I, Academic Press, New York, 1966. MR 36:840

5. D. Preiss, Limits of approximately continuous functions, Czechoslovak Math. J. 21 (1971), 371-372. MR 44:4154

6. W. Rudin, Principles of mathematical analysis, third edition, McGraw-Hill Inc., New York, 1976. MR 52:5893

7. Z. Zahorski, Sur la premiere derivee, Trans. Amer. Math. Soc. 69 (1950), 1-54. MR 12:247c

Department of Mathematics, University of Louisville, Louisville, Kentucky 40292

E-mail address: ubdarj01@homer.louisville.edu 